\title{
¿Qué piensan los profesores de las TIC?
}

Carmen Nolasco Salcedo

\section{Resumen}

En este artículo se pretende dar a conocer y entender la percepción que tienen los docentes, en el Centro Universitario de la Ciénega de la Universidad de Guadalajara, respecto al uso de las Tecnologías de la Información y la Comunicación (TIC), para proponer desde la comunicación estrategias efectivas que propicien el incremento de su uso como herramientas didácticas.

Palabras clave: TIC, docentes, educación, centro universitario, Moodle.

\section{WHAT DO TEACHERS THINK OF ICT?}

\begin{abstract}
In this article we want to explore and communicate the teachers' perception on Information and Communication Technologies (ICT) at Centro Universitario de la Ciénega from the University of Guadalajara, in order to propose effective strategies that promote its use as didactic tools.
\end{abstract}

Keywords: ICT, teachers, education, university center, Moodle. 


\section{Carmen Nolasco Salcedo}

Profesora de tiempo completo en la Universidad de Guadalajara, Centro Universitario de la Ciénega. Informática de formación, cuenta con estudios de maestría con mención en Programación por parte Universidad Martha Abreu de las Villas, Cuba, y Doctorado en Educación por parte de la Universidad Santander en Tamaulipas.

En el campo de la programación, ha trabajado en un proyecto denominado Olimpiadas del Saber. Sus temas de investigación son el impacto de la programación en preescolar, educación básica y media superior, y las tecnologías de la información y comunicación aplicadas a la educación superior.

\section{Introducción}

Ante la creciente evolución de las Tecnologías de la Información y la Comunicación (TIC), el Centro Universitario de la Ciénega ha incrementado las herramientas didácticas apoyadas en éstas, dando con ello un giro orientado a un cambio de paradigma, al facilitar el acceso y tratamiento de la información, y al reconocer las formas en cómo las tıc se pueden utilizar como apoyo en el proceso enseñanzaaprendizaje.

Las TIC representan un paso hacia la creación de nuevas formas de comunicación, ya que son herramientas dinámicas síncronas y asíncronas que facilitan la emisión, acceso y tratamiento de la información. La incorporación de estas tecnologías en la docencia conlleva a una nueva modalidad del proceso de enseñanza-aprendizaje. En este sentido, la Universidad de Guadalajara se ha propuesto incorporar las nuevas tecnologías educativas y con este fin inició un proceso de inversión-modernización en las Tıc, a fin de perfilar dicha institución hacia una nueva cultura de la calidad y aumentar la competitividad académica. Para ello, ha modificado su naturaleza y quehacer en procesos de planeación, organización académica y evaluación, con el propósito de adecuarse a los retos ocasionados por la actual sociedad del conocimiento, donde ahora las tıc siguen el ritmo de los continuos avances científicos en un marco de globalización económica y cultural. 
Imagen 1. Centro Universitario de la Ciénega.

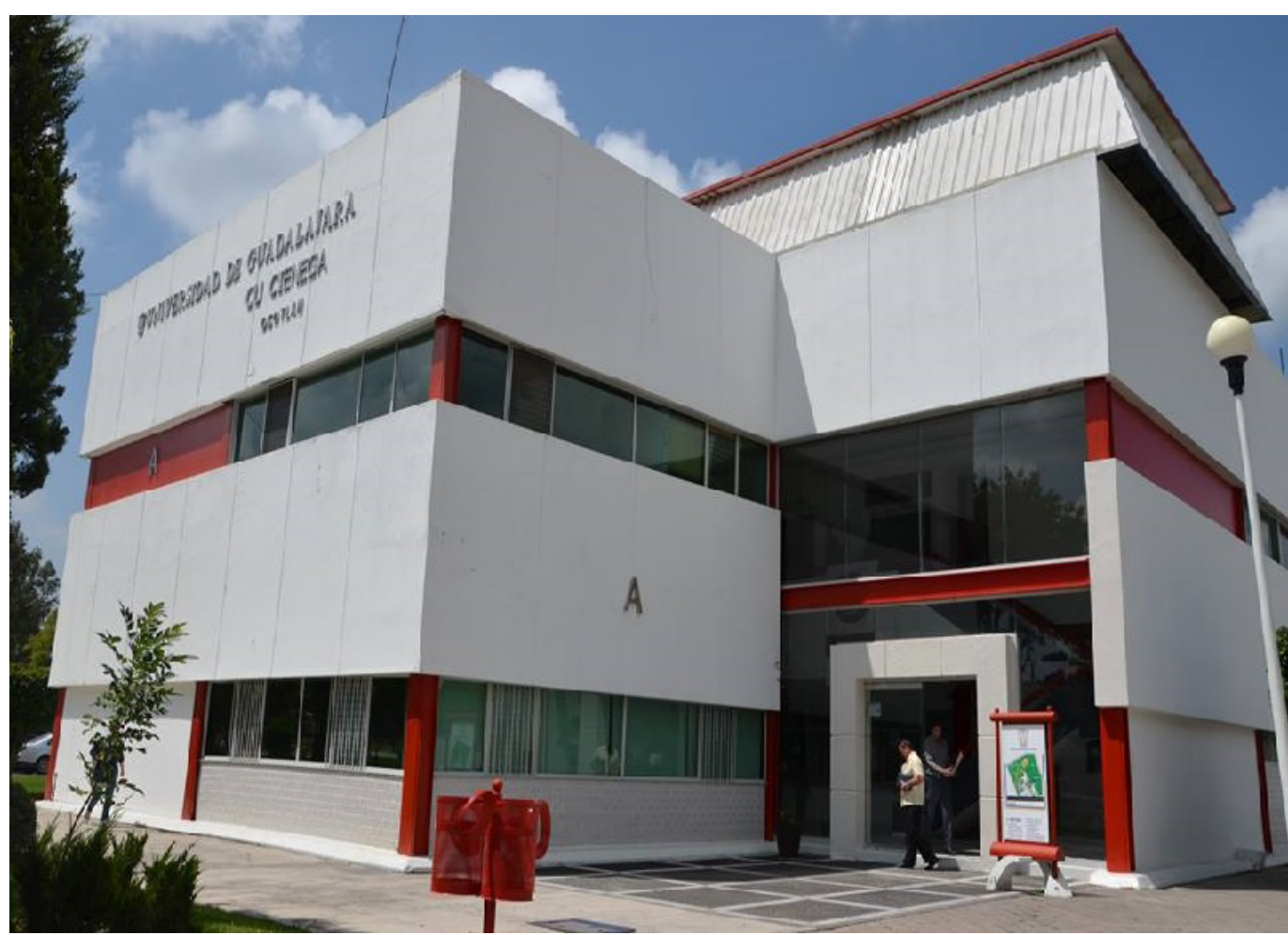

El Centro Universitario de la Ciénega (ver imagen 1) surgió en 1994, a través de tres sedes en donde se construyó infraestructura muy variada para el desarrollo de las funciones académicas: Ocotlán, La Barca y Atotonilco El Alto. La transformación de este centro universitario en la actualidad ha sido relevante, pues cuenta con una infraestructura idónea e integral que justifica la posición de vanguardia del Centro Universitario de la Ciénega por la calidad de sus instalaciones y planta académica.

Como parte del proceso de modernización, la Universidad de Guadalajara crea el Centro Universitario de los Valles (cu Valles) y el Centro Universitario del Norte (cu Norte), los cuales nacen con un modelo académico que trata de adecuarse a los requerimientos de flexibilidad y de apoyo en las tic. Con ello, la Universidad de Guadalajara se compone de seis centros universitarios metropolitanos y nueve centros universitarios regionales; la misión de cada uno es formar profesionales de calidad que mantengan un elevado sentido de responsabilidad social, que sean creativos y que puedan solucionar los problemas a los que se enfrentan con una visión sólida y equilibrada por el respeto a los valores y manteniendo un compromiso con el desarrollo local y regional de nuestra sociedad.

En el año 2000, los centros universitarios cu Valles y cu Norte comenzaron sus actividades académicas, con instalaciones y equipamiento tecnológico necesarios, además de un innovador modelo académico-pedagógico, que implicaba diferentes estrategias tales como capacitación al personal docente 
en ambientes virtuales, para que contaran con el perfil académico pertinente, material instruccional en línea, asesoría presencial y virtual, entre otras. cu Valles y cu Norte han crecido y progresado con un cúmulo de experiencias y desafíos, han logrado ser un componente dinámico que aporta conciencia crítica, conocimiento autónomo, formación profesional, tecnologías y cultura a la sociedad.

\section{El caso del Centro Universitario de la Ciénega}

El Centro Universitario de la Ciénega atraviesa tiempos de cambios propiciados por el desarrollo de las TIC, que han transformado las relaciones sociales y que a su vez influyen en la relación tecnología-educación. Se ha intentado incorporar de forma gradual las TIC en la educación, por lo que se han ofrecido cursos de capacitación al personal académico, con la finalidad de que conozcan los beneficios que éstas aportan a su labor docente, para que luego las utilicen como apoyo en el proceso de enseñanza-aprendizaje. No obstante, aun cuando los docentes tratan de adaptarse a las TIc, tienen muy arraigada la idea de que la enseñanza tradicional es un mejor método.

Dado que el papel de los profesores es fundamental para la efectividad del proceso de apropiación de las TIC y con el fin de que el Centro Universitario de la Ciénega esté un paso más cerca de alcanzar las condiciones favorables para su uso pedagógico, decidimos investigar la percepción que tienen los docentes acerca de las TIc y del cambio institucional que generan, especialmente en los programas educativos de Ingeniería en Informática e Ingeniería en Computación, que se relacionan directamente con dichas tecnologías.

Para profundizar apropiadamente sobre las diferentes percepciones y experiencias de los docentes respecto a las TIC en el Centro Universitario de la Ciénega se empleó un enfoque mixto. Se utilizaron dos técnicas para la recolección de datos: un cuestionario y una entrevista semiestructurada. A continuación, se describe cada una de ellas.

\section{Cuestionario}

El cuestionario consta de varios apartados:

1. Información general del docente. Recolección de situación profesional en donde están implicadas características tales como sexo, edad, puesto actual y años de experiencia.

2. Actividades prácticas docentes. Este apartado se refiere al recorrido profesional en la Universidad, incorporando las TIC en la práctica docente. Incluye preguntas tales como frecuencia del uso de las TIC en las diversas actividades docentes, si se consideran las tıc como apoyo fundamental en la docencia, y razones para incorporarlas en su práctica.

3. Opinión sobre las tıc e implicaciones para el docente. Incluye propuestas de mejora respecto a la apropiación de las mismas. 
Tabla 1. Distribución de la muestra por grupos de edad.

Tabla 2. Distribución de la muestra por experiencia profesional.

\begin{tabular}{|ccc|}
\hline Rango-Edad & Frecuencia & $\%$ \\
\hline 32-38 años & 10 & 19.6 \\
39-42 años & 12 & 23.5 \\
$43-46$ años & 15 & 29.4 \\
$47-50$ años & 9 & 17.6 \\
51-55 años & 5 & 9.8 \\
Total & 51 & 100.0 \\
\hline Antigüedad & Frecuencia & $\%$ \\
\hline 9-15 años & 25 & 49 \\
$16-18$ años & 11 & 22 \\
19-21 años & 8 & 16 \\
22-24 años & 6 & 12 \\
25-27 años & 1 & 1 \\
\hline
\end{tabular}

\section{Entrevista}

Se realizaron 5 entrevistas semiestructuradas, a 3 hombres y 2 mujeres. En ella se trataron las condiciones de organización de la Universidad respecto a la capacitación y/o formación en TIC, satisfacción personal a lo largo de la trayectoria como docente, y problemas y limitaciones para la incorporación de las Tic en el aula. De esta técnica de recolección de datos surge un análisis interpretativo en torno a las percepciones del docente respecto a la transformación del mismo como facilitador y las implicaciones que conllevan a ser parte de la revolución tecnológica en el ámbito educativo.

\section{¿Qué piensan los docentes de las TIC?}

De manera general, el personal docente de los programas educativos Ingeniería en Informática e Ingeniería en Computación del Centro Universitario de la Ciénega (93.8\%) afirma que está consciente de que las Tıc son una herramienta necesaria 
y que son muchas sus aportaciones en el proceso de enseñanza-aprendizaje en este modelo educativo; pero también señala, por un lado, que la incorporación de las TıC no garantiza per se que la educación sea mejor; 95\% considera que requiere mayor motivación por parte de la institución para convencerse de que estas herramientas son portadoras de una mejora en la calidad de la educación, de que realmente vale la pena si no de dejar totalmente el modelo tradicional de enseñanza, sí combinar ambos modelos y convertirlos en un modelo de educación optimizado.

En este mismo orden de ideas, 84\% de los docentes señala que es el catedrático quien con su práctica determina la calidad didáctica-pedagógica durante el proceso de enseñanza-aprendizaje (tabla 3). El que aumente la interacción profesorado-estudiante, según Sanabria y Hernández (2011, pp. 288), "depende de dos cuestiones importantes: las actividades de enseñanzaaprendizaje que genere el docente y la actitud del propio profesor hacia la expresión del estudiante". Así, sería también "precisamente a través de la apropiación como el individuo hace suyas las herramientas tecnológicas para utilizarlas en su relación con los escenarios socioculturales y contextos de actividad en los que se desenvuelve" (Colás y Jiménez, 2008, p. 196). Por lo anterior, es necesario que la institución educativa realice aportes específicos en la formación de docentes en lo que respecta al uso de las tic y a su implementación en el aula.

Tabla 3. Percepción respecto a la incorporación de las TIC en la práctica docente.

\begin{tabular}{|c|c|}
\hline $\begin{array}{c}\text { ¿Consideran las TIC como apoyo fundamental en la práctica } \\
\text { docente? }\end{array}$ & $\%$ \\
\hline $\begin{array}{l}\text { Son una herramienta necesaria y son muchas sus aportaciones en el } \\
\text { proceso de enseñanza-aprendizaje en este modelo educativo }\end{array}$ & 93.8 \\
\hline $\begin{array}{l}\text { Se requiere mayor motivación por parte de la institución para } \\
\text { convencerse de que estas herramientas son realmente portadoras } \\
\text { de una mejora en la calidad de la educación }\end{array}$ & 95.0 \\
\hline $\begin{array}{l}\text { El catedrático es quien con su práctica determina la calidad } \\
\text { didáctica-pedagógica durante el proceso de enseñanza-aprendizaje }\end{array}$ & 84.0 \\
\hline
\end{tabular}

Asimismo, 74\% del profesorado se esfuerza en integrar las TIc en la enseñanza, pero no ve recompensado su trabajo en la actitud de los jóvenes, debido a que los estudiantes se interesan más por el contacto directo con el profesor, lo que implica la necesidad de fortalecer en aquellos los procesos educativos independientes que ayuden a desarrollar otras potencialidades.

En opinión de $72.5 \%$ de los profesores, la comunidad estudiantil prefiere sus clases en el aula, donde el docente es transmisor y el alumno receptor (tabla 4). Sin embargo, como señalan Olcott y Scmidt, "el profesorado ya no es guardián del conocimiento... El papel que un docente deberá desempeñar en un futuro puede ser parecido al de un director de orquesta: por un lado, deberá motivar, dirigir y dar autonomía a los músicos (el alumnado) y además deberán cuestionar, facilitar y crear un todo que sea más que la suma de los estudiantes individuales" (2002, pp. 269-270). 
Tabla 4. Percepción respecto a la incorporación de las TIC en la práctica docente.

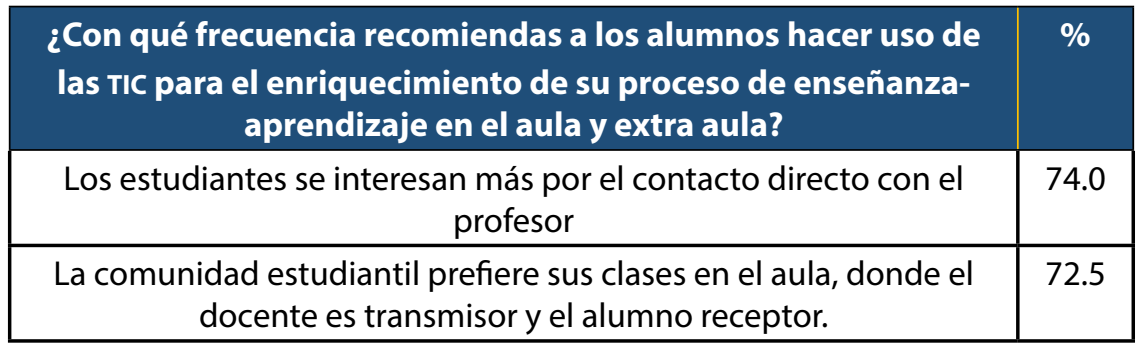

Habría que comprobar que, efectivamente, la comunidad estudiantil tiene dicha percepción. De ser así, es menester capacitarla e informarla respecto a las ventajas que las tic proporcionan a su proceso de aprendizaje y las habilidades que redescubrirán en sí mismos. Lograr que los estudiantes reconozcan que las TIC no son sólo un espacio para jugar, charlar y pasar el tiempo.

Cabe mencionar que los docentes tienen diferentes alternativas para innovar en el aula, co-construyendo aprendizaje significativo, de dirigir la mirada a otras herramientas como Facebook, Twitter, YouTube, que si bien no fueron creadas precisamente para la actividad educativa sí se han vuelto espacios para compartir materiales académicos con los estudiantes, debido a que éstos pasan gran parte de su tiempo conectados en dichas redes sociales. Es necesario crear espacios de diálogo, de debate, para involucrar a los alumnos en la actividad formativa, en el conocimiento de los beneficios que se producen en el contexto, insertándolos en las redes sociales para lograr un aprendizaje activo en el cual todos los miembros del grupo colaboren en la construcción del conocimiento y contribuyan al aprendizaje de todos.

Los profesores piensan que es necesario que su institución fomente estrategias que los motiven a apropiarse de las TIC: 91.3\% de los encuestados considera importante que haya una mejora en el reconocimiento de la labor docente, $85.1 \%$ propone intercambio de experiencias entre ellos, $81.4 \%$ coincide en que se creen espacios para compartir prácticas docentes y $73.3 \%$ expresa la necesidad de acciones formativas que incidan en los docentes (tabla 5).

\begin{tabular}{|l|c|}
\hline \multicolumn{1}{|c|}{ Propuestas de mejora respecto a la apropiación de las TIC } & $\%$ \\
\hline Reconocimiento de la labor docente & 91.3 \\
\hline Intercambio de experiencias docentes & 85.1 \\
\hline Crear espacios para compartir prácticas docentes & 81.4 \\
\hline Acciones formativas que incidan en el personal docente & 73.3 \\
\hline
\end{tabular}

Con las encuestas también se encontró que la mayoría de los docentes (58\%) ha conocido las herramientas de las Tıc por iniciativa propia, lo que expresa el interés de utilizarlas como apoyo en el aula; otro grupo significativo (42\%) conoció
Tabla 5. Propuestas de mejora respecto a la apropiación de las TIC. 
Tabla 6. Noción respecto a las herramientas que ofrecen las TIC. las herramientas a través de cursos de capacitación, lo cual puede reflejar cuán interesados están los profesores en emplear tecnologías que apoyen los procesos educativos (tabla 6).

De esta manera, es evidente que la falta de formación en Tic es la razón principal para que tanto el personal docente como la comunidad estudiantil se resistan al cambio de rol que implica esta nueva era del conocimiento. Lo anterior está en concordancia con lo que plantean Roa y Stipcich, que "en ocasiones, debido a la falta de información, la informática se percibe como una amenaza, un desafío, una innovación más, manteniendo el profesor una actitud escéptica frente a las potenciales mejoras que puede aportar" (2009, p. 166).

\begin{tabular}{|l|c|}
\hline $\begin{array}{c}\text { ¿De qué forma conoció las herramientas que ofrecen las TIC en el ámbito } \\
\text { educativo? }\end{array}$ & $\%$ \\
\hline a través de cursos de capacitación & 42 \\
\hline Iniciativa propia & 58 \\
\hline
\end{tabular}

\section{TIC utilizadas entre los docentes universitarios}

Según las encuestas, se observó que de las herramientas que las tıc proporcionan para la generación de conocimiento, las más utilizadas por el personal docente son los motores de búsqueda, presentación de información a través de videos y PowerPoint para las exposiciones orales. Para la comunicación usan el correo electrónico y las redes sociales, más específicamente Facebook para resolver dudas o para envíos de trabajos. Algunos profesores mencionaron que utilizan podcast y blogs.

Se pudo determinar que más de la mitad de los encuestados (85\%) conocía Moodle y de éstos 30\% ya tenía su curso publicado en dicha plataforma, ya sea en desarrollo o activo para la comunidad universitaria, mientras que 15\% la utiliza sólo para aplicar exámenes. Los docentes ven esta plataforma como un espacio a través del cual se puede beneficiar el proceso educativo en cuestiones de tiempo, además de convertirse en el punto de referencia de los estudiantes para consultar y adelantar los contenidos de la clase y útil como una herramienta de apoyo para la aplicación de exámenes y actividades. No obstante, hay quienes consideran este tipo de plataformas como mecanismos muy cerrados y poco utilizables, ya que, en primer lugar, sólo permiten la interacción entre los usuarios que tienen una cuenta y clave para acceder; y, en segundo lugar, les parece un espacio poco amigable, donde se requiere la habilidad de la creatividad para lograr resultados satisfactorios. Además, los estudiantes no están tan familiarizados con este tipo de plataformas como con las redes sociales, de ahí que algunos docentes prefieran publicar sus contenidos en sitios más públicos, para que también se pueda incluir a quienes no son parte del proceso educativo formal. 


\section{Percepción de las políticas universitarias para la integración de TIC}

Riascos-Erazo, Quintero-Calvache y Ávila-Fajardo (2009, p. 137) manifiestan dos perfiles acerca de la interacción de los profesores con las innovaciones en el proceso enseñanza-aprendizaje: aquellos docentes que se resisten a involucrase con los nuevos instrumentos o formas de enseñar, aduciendo que los métodos que han utilizado durante tanto tiempo han funcionado bien y que no consideran necesario hacer cambios, y aquellos otros que, con mentalidad más abierta, están dispuestos a integrarse en este rol de docente innovador, en busca de mejorar las técnicas existentes de aprendizaje y planteamiento de nuevos ideales pedagógicos.

De esta manera, la actitud de los docentes respecto al uso de las tic difiere mucho, pero, debido al esfuerzo del Centro Universitario, la percepción ha ido mejorando, manifestándose en un aumento en la utilización de las mismas. Sin embargo, se deben seguir haciendo gestiones por parte de las instituciones para que la enseñanza tradicional en la práctica docente se cambie por una modalidad educativa apoyada en el uso de las TIc. Así también lo consideran los docentes: de acuerdo con ellos, para poder aceptar el cambio de rol que implica esta modalidad es necesario que el Centro Universitario mantenga un elevado sentido de responsabilidad académica, con un firme compromiso con los docentes, proporcionándoles diferentes estrategias que coadyuven a potenciar los mecanismos que se emplean actualmente a través del Programa Integración de las tic a la docencia. De esta manera, el papel que juega el Centro Universitario de la Ciénega en el proceso del uso de las tic como herramientas de apoyo a la enseñanza-aprendizaje debe ser dinámico y motivador respecto a la actividad académica, impulsando a que el docente haga uso de las herramientas tecnológicas en su quehacer educativo, para que se inserte en el proceso de transformación institucional.

Imagen 2. Aula de estudio: docente, en el proceso de enseñanzaaprendizaje del estudiante.

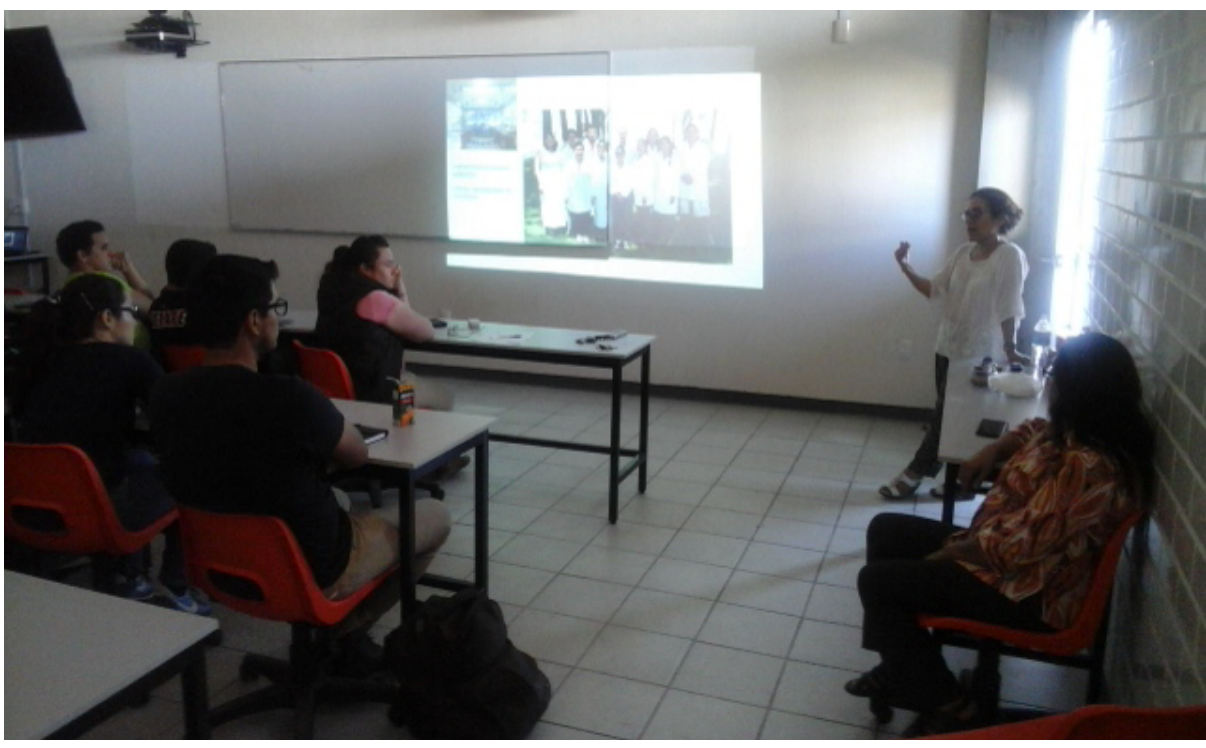




\section{Conclusiones}

El Centro Universitario de la Ciénega ha ampliado su infraestructura tecnológica (ver imagen 3) considerando las TIC como herramientas para el apoyo a los procesos de enseñanza-aprendizaje, que permiten una conformación, dinámica y colaborativa entre los participantes, donde se facilitan instancias de evaluación formativa permanente respecto al proceso de aprendizaje del alumnado.

Imagen 3. Módulo de Tutorías e Investigación.

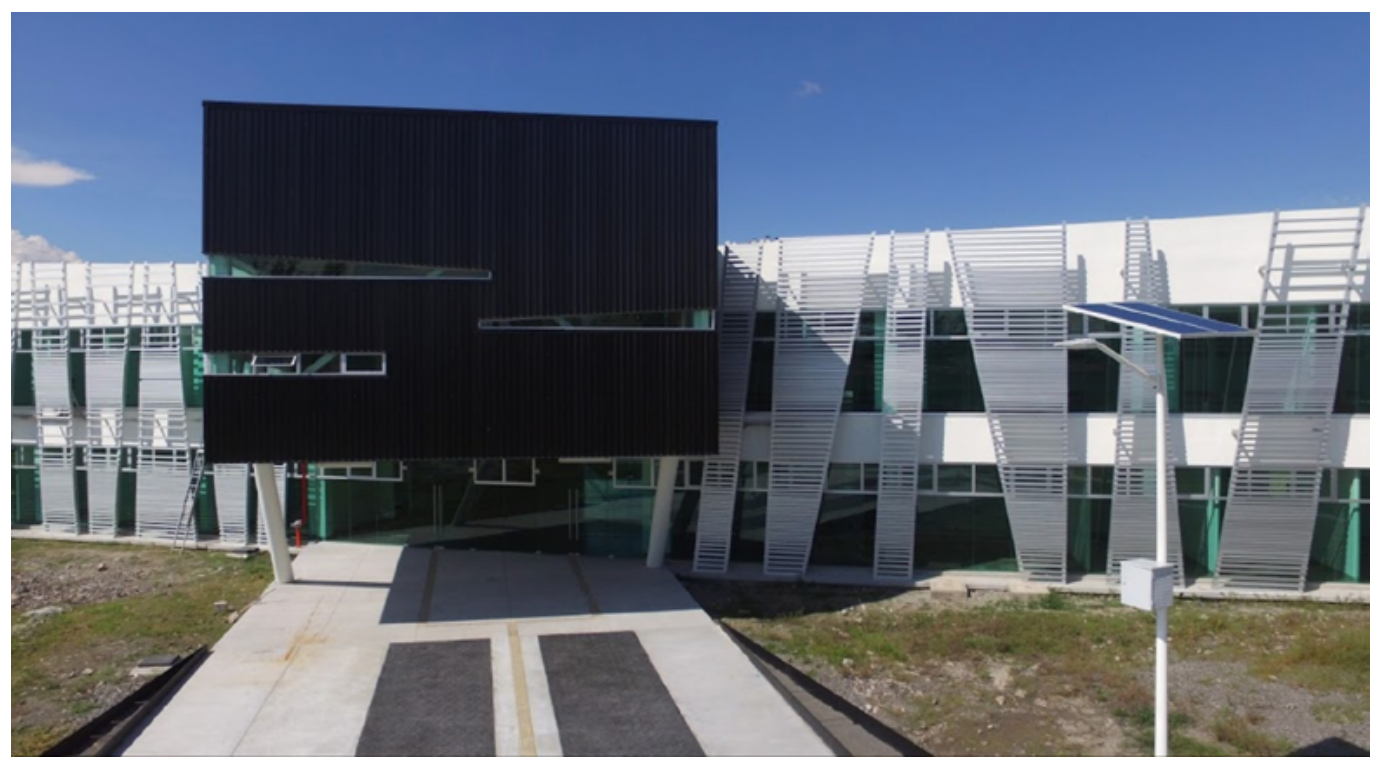

No obstante, la tendencia en el empleo de las tıc entre los docentes universitarios sigue siendo limitada a la búsqueda y presentación de contenidos, ya que, de momento, su uso no ha promovido cambios substanciales en los procesos educativos en curso y en la mayoría de los casos se siguen utilizando herramientas tradicionales. Cabe mencionar que cuando se introducen las tic en el aula, las actividades dirigidas por el profesor disminuyen en beneficio de las actividades independientes y cooperativas, el enfoque didáctico se inclina hacia uno más constructivista y los estudiantes tienden a integrar el pensamiento verbal y visual.

El personal académico todavía se resiste al cambio, sobre todo aquellos que han sido formados con el modelo tradicional y consideran que ese es la mejor opción para seguir impartiendo sus clases en el aula. Es por esto que es necesario contar con personal capacitado que muestre a los profesores cómo las tıc pueden volverse aliadas para fortalecer los procesos de enseñanzaaprendizaje. En este contexto, el Centro Universitario debe establecer estrategias didácticas para presentar las ventajas de las TIC como herramientas de apoyo en el proceso de enseñanza-aprendizaje a los profesores que más se resisten al cambio (ver imagen 4).

De la misma forma, es necesario que haya una inclusión por parte de los alumnos, que éstos se vayan adaptando a las herramientas que ofrecen 
Imagen 4. Profesores en el programa de formación académica. ambientes virtuales con actividades y recursos realmente innovadores y que puedan entender poco a poco las potencialidades que esta práctica puede tener para el proceso formativo. Por lo tanto, la utilización de las Tıc en el quehacer educativo implica formación en la incorporación de las Tic tanto a personal docente como a estudiantes, así como el establecimiento de estrategias educativas que aumenten la motivación y disminuyan la brecha generacional entre ambos.

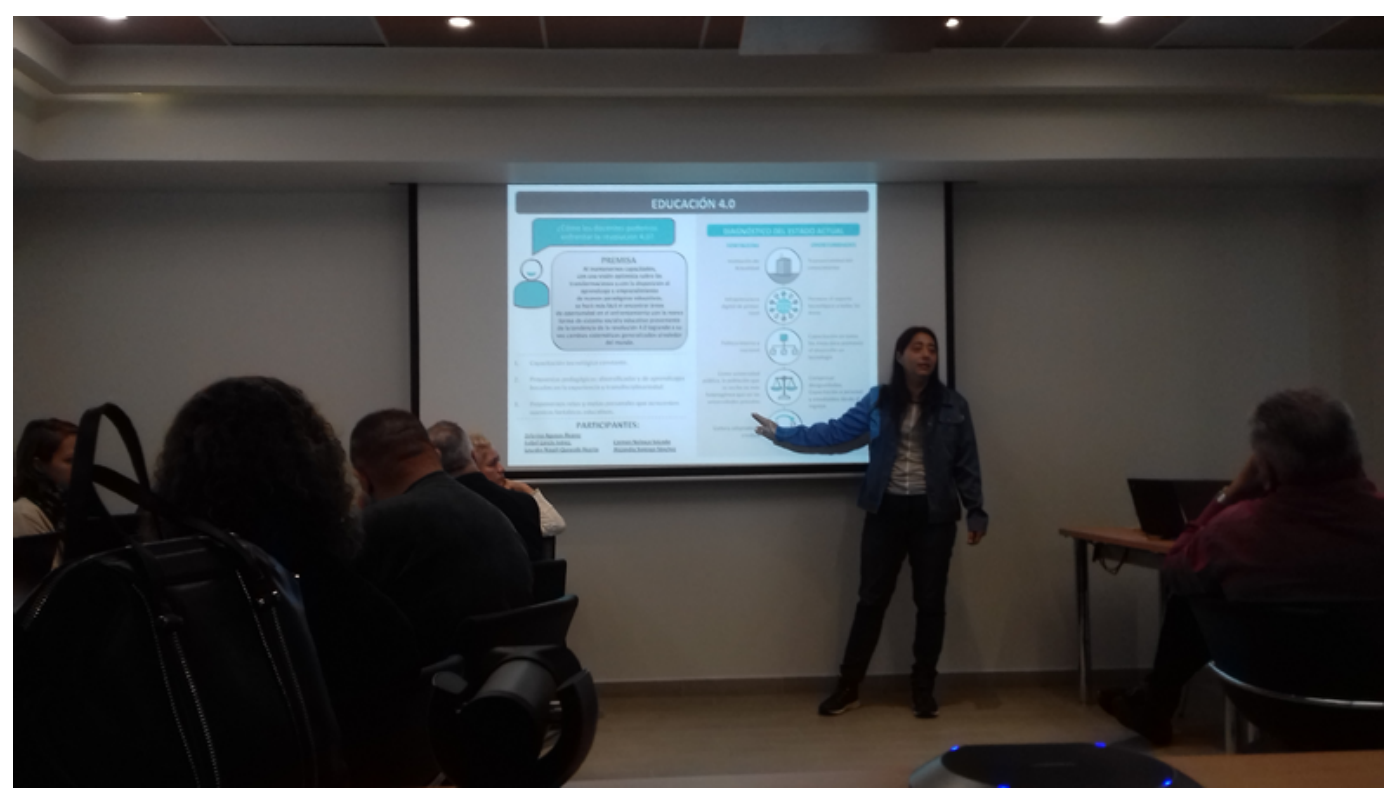

Se debe fomentar, asimismo, la comunicación en este tipo de procesos, pues sensibiliza, informa y genera redes colaborativas que permiten que la apropiación de las tecnologías tenga una verdadera repercusión positiva en el sistema educativo.

\section{Recomendaciones}

El uso de las Tic en la práctica docente no se adquiere de la noche a la mañana ni con sólo un curso de capacitación. Es un proceso lento, gradual y lleno de altibajos. Por ello, se recomienda al docente que quiera iniciar una experiencia de uso de las Tic en el aula que planifique adecuadamente, que no improvise y que sea consiente de los problemas y dificultades técnicas que puedan surgir.

Otro factor importante para el uso de las tic en el aula es la necesidad de formación del personal docente. Los profesores deben ser los primeros en usar las tecnologías y deben de dominarlas totalmente antes de incorporarlas a su proceso de enseñanza.

El profesorado debe saber analizar y perfeccionar su práctica educativa, trabajando colaborativamente con colegas en proyectos comunes basados en una formación permanente y en la reflexión de la práctica educativa, como 
requisitos fundamentales para el desarrollo de procesos de enseñanza adecuados a los nuevos contextos y entornos tecnológicos.

Se debe potenciar el trabajo colaborativo mediante las TIC, al participar en comunidades de aprendizaje donde todos se beneficien del conocimiento del resto de los miembros participantes.

\section{Referencias}

* Colas, P., Jiménez R. (2008). Evaluación del impacto de la formación (online) en TIC en el profesorado. Una perspectiva sociocultural. Revista de Educación, 346, mayo-agosto 2008, 187-215. Recuperado de: http://www.revistaeducacion.mec. es/re346/re346 07.pdf.

* Riascos-Erazo, S., Quintero-Calvache, D. y Ávila-Fajardo, G. (2009). Las TIC en el aula: percepciones de los profesores universitarios. Educación y Educadores, 12 (3), 133-157. Recuperado de: http://w.redalyc.org/articulo.oa?id=83412235008.

* Roa, M. y Stipcich, M. S. (2009). Los docentes en relación con las tecnologías. Recuperado de: http://revistas.usal.es/index.php/eks/article/view/15726/16228.

* Moscovici, S. (1997). Social Representations Theory and Social Constructionism. Recuperado de: http://psyberlink.flogiston.ru/internet/bits/mosc1.htm.

* Sanabria, A., y Hernandez, C. (2011). Percepción de los estudiantes y profesores sobre el uso de las TIC en los procesos de cambio e innovación en la enseñanza superior. Revista Aloma, 29, 2011, 273-290. Recuperado de: http://www. revistaaloma.net/index.php/aloma/article/view/106/82.

\section{Cómo citar este artículo}

* Nolasco Salcedo, Carmen (2019). ¿Qué piensan los profesores de las TIC? Revista Digital Universitaria (RDU). Vol. 20, núm. 2 marzo-abril. DoI: http://doi.org/10.22201/ codeic.16076079e.2019.v20n2.a6 